\title{
MODELLING LIFESTYLE ASPECTS INFLUENCING THE RESIDENTIAL LOAD-CURVE
}

\author{
Wolfgang Hauser \\ Stuttgart University \\ ZIRN \\ Seidenstr. 36, 70174 Stuttgart, Germany \\ wolfgang.hauser@sowi.uni-stuttgart.de \\ José Évora \\ University of Las Palmas de Gran Canaria \\ Parque Científico y Tecnológico \\ 35017 Las Palmas de Gran Canaria, Spain \\ jose.evora@siani.es \\ Enrique Kremers \\ EIFER \\ Emmy-Noether-Str.11, 76131 Karlsruhe, Germany \\ enrique.kremers@eifer.uni-karlsruhe.de
}

\section{KEYWORDS}

ABS. Agent-based-model, residential energy consumption, load-curves, lifestyle.

\begin{abstract}
Using the results of a representative survey for the city of Stuttgart, household load-curves are simulated through an agent-based modelling approach. Aggregated household load-curves for different lifestyle groups are presented and the effect of their corresponding behavioral profiles on the residential load-curve is evaluated.
\end{abstract}

\section{INTRODUCTION}

Residential consumption of electricity is influenced by a multitude of variables and shows big variance between households, even within the same society and geographic region: Lutzenhiser and Bender (2008) report differences of up to factor 40 between the measured electricity demands of 1627 households in a Northern Californian sample Morley and Hazas (2011). Furthermore, electrical consumption for single household tasks vary greatly between households: ADEME et al. (2008) shows that electricity used for cooling devices differs by factor 10 between different households, the same applies to electricity used per person for dish-washers.

The rise of decentralized power supply raises the need for electricity demand forecasts of smaller areas. Simulations of household electricity demand are mostly based on mean values of the whole population (e.g. Paatero and Lund (2006)); for specific areas of interest this approach results in an ecological fallacy, because different kinds of households are not equally distributed in space: In Stuttgart the average number of persons per household differs from 1.56 to 2.18 for different city quarters. To reduce the ecological fallacy it is necessary to identify determinants of residential electricity consumption, which can be linked to geographic data or building types, e.g. the number of persons living in a household or lifestyle typologies.

For the planning of power grids, not only the overall quantity of electricity consumed is of importance, but it is also important to know at what time of day the electricity is demanded: it is the load curve that matters (a load curve visualizes the use of electrical energy over time, showing watts on the y-axis and time on the $\mathrm{x}$-axis). Nevertheless, measured data about electricity demand on a household level is very hard to find, especially when looking for a random sample. To simulate load curves for different types of households, we connected a simulation converting weekly or daily probabilities of energy relevant household tasks, into start times of events with simulations of appliances' load curves.

\section{METHODOLOGY}

Based on the lifestyle typology developed by Otte (2005) usage rates and probabilities for the possession of different electrical appliances are compared between different groups of society, using data collected in a postal survey. In order to evaluate household energy consumption, the survey focused specifically on daily routines and appliance ownership, because direct data collection of household energy con- 
sumption through surveys faces a big problem regarding missing values (25-60\% in most studies), which can not be expected to be distributed randomly. Usage rates, habits and probabilities for the possession of household equipment are transformed into household-agents with specific daily routines and specific household equipment, then the resulting load curves for each household are simulated in an agent based model and aggregated in different ways.

\section{DATA}

We get information about weekly and daily usage rates for different appliances from a postal survey conducted in the run of the European Centers and Laboratories for Energy Efficiency Research (ECLEER) Ph.D program. In Germany, 4000 Stuttgart based households had been asked to fill out a questionnaire about household usage rates, sociodemographics and lifestyle issues; 769 filled out questionnaires have been sent back, equalling a return rate of $19.2 \%$. Results of the survey can be compared with a survey employing the same typology, conducted in 2008 by the Statistisches Amt of the city of Stuttgart, with a bigger sample and a better return rate Schwarz (2010). Our results are more or less in line with this survey, with the exception of a higher age average resulting in smaller proportions of modern lifestyles. The biggest differences are with regard to the proportion of Conventionalists and Hedonists. It seems plausible that younger people, spending less time at home, were less willing to take the time to answer our questionnaire, as it was substantially longer than the one used by Schwarz (2010), which also explains the lower overall return rate. A comparison of the results of both surveys is presented in Table 1.

\section{SIMULATION MODEL}

After the survey analysis, the information regarding the appliances usage for every social group is available. Now, the challenge is to build up a simulation where this information is integrated in order to calibrate an agent-based model. However, this task concerns external aspects which provide the agents environment. The environment is composed of two sides: the global and the local. The global regards the agent location within the world. This information arises when the agent is related to a concrete household of the scene and this is, in turn, related to a building. The first relation is due to a link and the second one is due to a contain relation. Finally, the building indicates the exact coordinates to locate itself in the world. On the other hand, the local environment consists of the appliances within the household as they are the elements with which the agent interacts.

Then, the simulation model describes agents, appliances, households and buildings. As described in Évora et al. (2011), the agents can be considered as intentional models and the appliances as design models. The agent model is described in section Household Model and represents parts of the behavior of a household, which have a big impact on consumption of electrical energy. The local environment of the agent is composed of many kinds of appliances which are switched by the agent. Then, the task to develop the local environment consists of modelling the many appliances that the agent uses. The appliances models consists in the description of how they work. For instance, the washing machine model works producing a non-consumption when switched off. When the agent turns the washing machine on, a three-cycle working mode starts up producing a non-zero consumption.

This model is represented using a bottom-up approach where the electrical grid consumption comes from the agents acts since they generate the appliances consumptions at switching them. The tool that we have used to model this experiment is Tafat (described in detail in Évora (2011)). This framework was used because, among other reasons, it allowed us developing the model fast since the appliances models reuse was possible. Another reason for choosing this framework was the performance as the intention is to model a huge scene where thousands of agents are running at the same time with their respective local and global environments.

\section{Household Model}

Each household is represented as an agent, having control over his electrical appliances; the probability to own a certain kind of appliance is derived from the distributions in the survey data and differs between the lifestyle groups (see section Survey Results). Each instance of an household draws randomly from the respective distribution, in order to determine if he owns a tumbler, washing machine, etc and what kind of cooling devices are to be found. In the same fashion, each household is assigned rates of using these appliances, as well as times of inactivity (sleep hours) and absence. Probabilities of 
Table 1: Otte lifestyle groups in Stuttgart

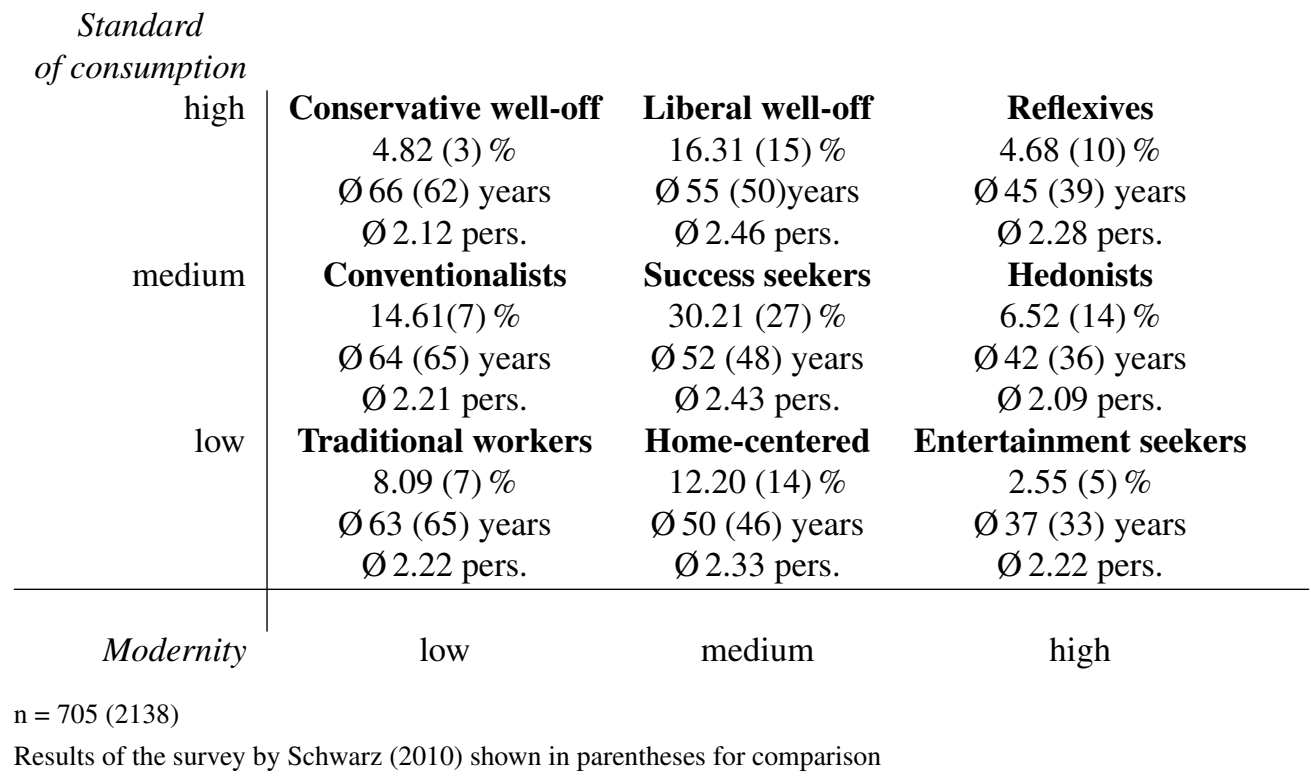

preparing a warm lunch or dinner and the time when these take place are also taken from the survey and differ between groups. Of course, the agent can not perform the household tasks in his time of absence or sleep. It is, however, able to start multiple devices at the same time, which will run for a predefined cycle (washing machine) or for a time that is, again, drawn from the distribution of the lifestyle group he belongs to. Lights are turned on automatically between 18:00 and 7:00 if the agent is not absent or sleeping; however, we added a normally distributed error component to the start and stop time, in order to prevent an artificial peak to the aggregated load-curve. Cooling devices are running on a regular pulse. To generate a load-curve that averages the behavior of the households of interest and is robust towards random variation, 1000 households are set up for each simulation run. The behavior model of the households is connected to the Tafat environment, controlling the start and stop times for the appliance models stored therein.

\section{Appliance Models}

Each household is equipped with a set of appliances. Besides the usage of the appliances, the electrical devices themselves have different types of consumption patterns - for example a lightning bulb will run most of the time at a constant power, whereas a washing machine will consume more power during the heating cycle than during the washing period. The consumption depends on the type of electrical consumer and on its internal mechanisms of operation. High power devices such as ovens or stoves will usually operate in an intermittent mode, causing high power peaks, separated by almost zero consumption periods.

For simulating the different types of devices, the European Institute for Energy Research (EIFER) and the University College of Engineering at the University of the Basque Country (EUI/UPV) have developed a set of appliance models which allow to represent the load curves of individual devices. Parametering the devices is also possible, in order to represent different efficient appliances of the same type, e.g. characterized by their EU energy label or size. The device models can be switched on and off in simulation time, as if they would be a real device, and generate a load which is aggregated to the household load.

\section{RESULTS}

\section{Survey Results}

Regarding the ownership of electrical appliances and daily routines, there are significant differences between lifestyle groups. Conservative well-off and 
Entertainment seekers appear to be specially suited for a direct comparison, as they are on the opposing ends of the lifestyle dimensions and show the biggest differences in appliance possession and usage rates. For this reason, these groups have been chosen for the simulation runs. A detailed analysis of all variables can not be presented in the scope if this article, so only the most striking differences will be presented.

In regard to the number of cooling devices to be found in households, Conservative well-off own on average 1.9 devices, meaning that almost every household owns two devices, while only one in three Entertainment seekers households has a second cooling device. A Welch t-test between the group shows that this difference between the two groups is significant on the $99.9 \%$-level. Furthermore, we find significant differences regarding the percentage of household owning a of dish-washer (85\% vs. $39 \%$ ) or a tumble dryer (53\% vs. $11 \%$ ). Regarding daily routines, the two groups differ significantly in the number of times they are cooking per week (8.0 vs. $5.5)$ and in regard to their absence from home (see Figure 1).

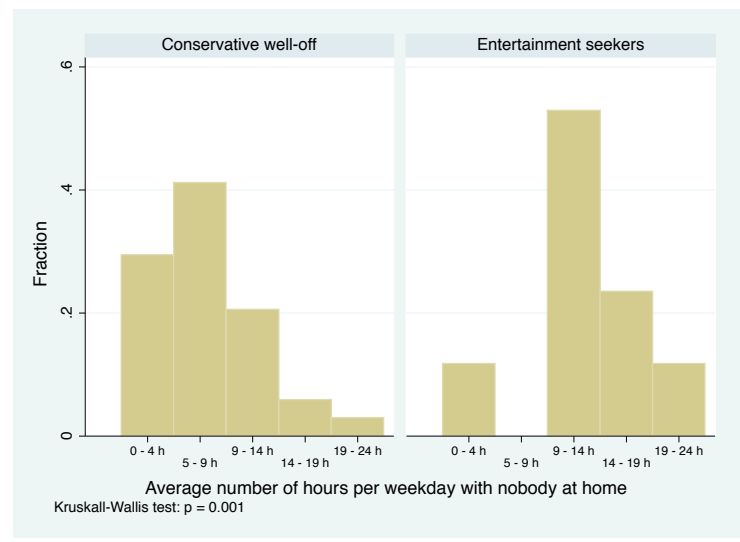

Figure 1: Absence from home on weekdays

\section{Simulation Runs}

Data about measured load-curves of different household is very sparse and not publicly available. This was one of the motivations for our approach. Unfortunately, this prevents a good verification of our results with measured data. Therefore, we compare the simulated load-curves (cumulatedSIM) with a standard household load-profile scaled to the electrical energy consumption of the simulation (weighted-
SLP). To evaluate the effect of the different lifestyles on the residential load-curve, we present results of three simulation runs: one with the share of the different lifestyle groups as found in the survey, one where all the households are set to the behavior and appliance ownership of the group of the conservative well-off and a last one with all households set to values found for the group of the entertainment-seekers.

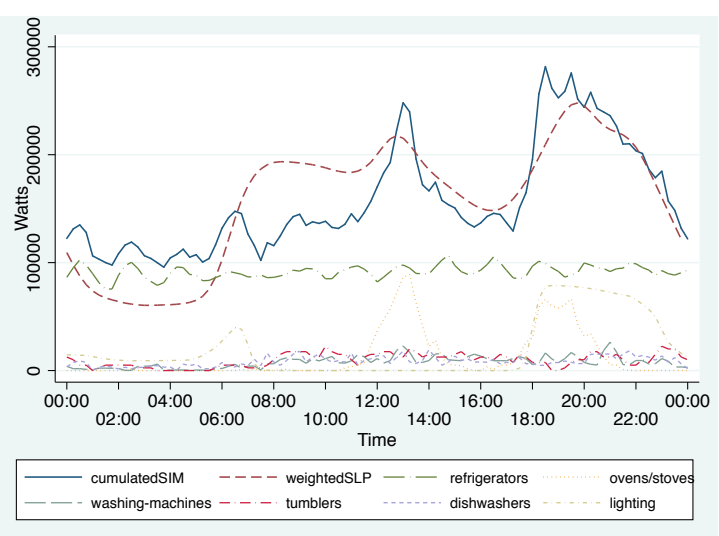

Figure 2: Simulated load-curve for 1000 households with lifestyle groups distributed according to their share in the survey

With lifestyles represented in the simulation with the same shares as in our sample (Figure 2), we see that the noon and evening peaks are more accentuated than in the standard household load profile (SLP). One reason for this seems to be, that we model only a relatively small number of appliances by now and therefore the effect of stoves and ovens could be overestimated. Another reason - which applies especially to the evening peak - might be that employment figures in Stuttgart are very high, so that more consumption than on average in Germany is shifted towards the evening hours. A third reason might be, that we underestimated the variation in lunch and dinner preparation and should introduce a bigger error component to this decision.

Another difference in comparison to the SLP is that the simulated load curve does not decline as deep in the night hours and rises less sharply in the morning. With the small number of devices which can be simulated at the moment, cooling devices which produce a continuous pulse - make up a big part of the electricity consumption in our simulation; even though refrigerators and freezers have a significant impact on the electricity consumption of 
a household, their smoothing effect on the simulated load curve might be exaggerated here. However, our aim was to present the opportunities of a simulation model based solely on survey data and theoretical considerations, before extensive calibration and the simulated load curve shows a clear resemblance towards the SPL.

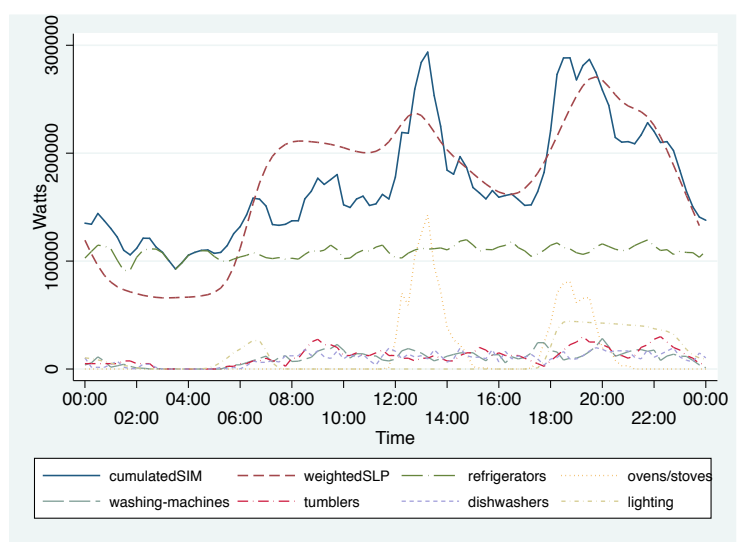

Figure 3: Simulated load-curve for 1000 households with the profile of the conservative well-off

When we compare the load-curve of the conservative well-off (Figure 3) with the standard load profile or with the load curves of the other simulation runs, the most striking difference is the very pronounced peak at the middle of the day. In this group we find more elderly people than in the other groups, which explains why they spend more time at home, giving them the opportunity to consume more electricity during work hours. The share of people having a warm lunch at home is bigger than in the other groups which raises the peak at the middle of the day to a higher level than in any other group. At the same time, the percentage of people having a cold meal for dinner is rather high in this group, which explains why the evening peak is relatively small here. Overall their load-curve is on a higher level than the others, because they live in more spacious apartments/houses and own more electrical appliances than i.e. the entertainment-seekers (see section Survey Results). Their ownership of many cooling devices and the habit of having a warm meal for lunch reported clearly shows in the simulated load curve.

The load-curve of the entertainment-seekers (Figure 4) is characterized by the absence of the peak in the middle of the day and by a lower level than

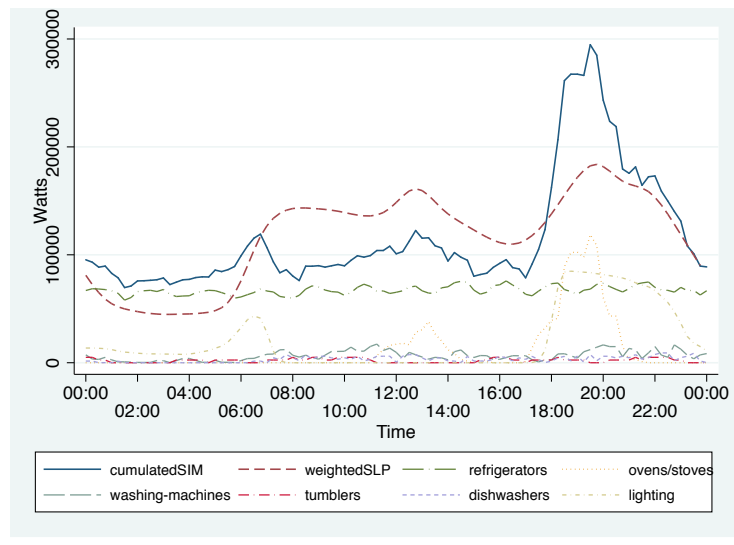

Figure 4: Simulated load-curve for 1000 households with the profile of the entertainment seekers

the other load-curves. Households belonging to this group are rather young and there are less retired persons to be found in this group than amongst the conservative well-off, which is one reason why they spend more time out of home than other groups. Their consumption is therefore shifted to the evening hours, where they create a larger peak than the other groups. They have relatively few household appliances like dishwashers, tumble dryers and washing machines which decreases their energy consumption significantly.

\section{CONCLUSIONS AND OUTLOOK}

The simulation shows that the different lifestyles clearly produce different load-curves, which correspond to their behavior and ownership of appliances, even though not all the survey results and all appliances to be found in a household could be modelled by now. We expect the differences between the lifestyle groups to rise with the progressing inclusion of further appliances, e.g. water heaters and further entertainment electronics. As lifestyles are not equally distributed in space, this fact is important when planning power grids, especially when thinking about decentralized power supply. The smaller the area which is supplied by a single power source, the more important these differences become. The ecological fallacy of assuming a standard average load-profile for all households inside a specific region could lead to a wrong estimation not only of the level of electricity demand, but also of the shape of the load-curve. 


\section{ACKNOWLEDGEMENTS}

The Tafat simulation framework is developed by the Instituto Universitario de Sistemas Inteligentes y Aplicaciones Numéricas en Ingeniería (SIANI). As a simulation framework, it joins many different simulation models by different authors and profited from advice of many colleagues at EIFER, SIANI and ZIRN, too numerous to name them all. Nevertheless, we want to thank at least some persons, without whom this article would not have been possible: we gratefully acknowledge the advice and insights from Oscar Barambones, Jose María Gonzalez de Durana, José Juan Hernández, Mario Hernández, Pablo Viejo and Wolfgang Weimer-Jehle.

\section{REFERENCES}

ADEME, ENERTECH, and EDF (2008). Campagne de mesures des appareils de production de froid et des appareils de lavage dans 100 logements. Technical report.

Lutzenhiser, L. and Bender, S. (2008). The "Average American" unmasked: Social structure and differences in household energy use and carbon emissions. In 2008 ACEEE Summer Study on Energy Efficiency in Buildings.

Morley, J. and Hazas, M. (2011). The significance of difference: Understanding variation in household energy consumption. In ECEEE 2011 summer study. Energy efficiency first: the foundation of a low carbon society.

Otte, G. (2005). Entwicklung und Test einer integrativen Typologie der Lebensführung für die Bundesrepublik Deutschland. Construction and test of an integrative lifestyle-typology for germany. Zeitschrift für Soziologie, 34(6):442-467.

Paatero, J. and Lund, P. (2006). A model for generating household electricity load profiles. International Journal of Energy Research, 30(5):273-290.

Schwarz, T. (2010). Lebensstile und Wählerverhalten in Stuttgart. Ergebnisse der Stuttgarter Lebensstilbefragung 2008. Statistik und Informationsmanagement, (7).

Évora, J. (2011). Tafat-Profiler: una herramienta para la generación automática de modelos de simulación a partir de perfiles. Technical report.

Évora, J., Kremers, E., Cueva, S., Hernández, M., Hernández, J., and Viejo, P. (2011). Agent-based modelling of electrical load at household level. In Stepney, S., Welch, P. H., and Andrews, P. S., editors, ECAL 2011: CoSMoS - Proceedings of the 2011 Workshop on Complex Systems Modelling and Simulation, page 15, Paris.

\section{AUTHOR BIOGRAPHIES}

WOLFGANG HAUSER graduated in sociology at the University of Bamberg, Germany. During his studies he has been working at the Institute for Employment Research, Nuremberg. In 2008 he joined the Interdisciplinary Research Unit on Risk Governance and Sustainable Technology Development (ZIRN) at Stuttgart University, where he is working on a Ph.D.-thesis inside the framework of the European Centre and Laboratories for Energy Efficiency Research (ECLEER) and on an agent-based simulation of the german electricity market (AMIRIS).

JOSE ÉVORA graduated in computer engineering at the University of Las Palmas de Gran Canaria, Spain in 2010. He started his research in energy efficiency and sustainability at SIANI in 2010. On April 2011, he received a grant from the Canary Agency of Researching, Innovation and Society Information to develop his Ph.D. On June 2011, he finished the master degree in Intelligent Systems and Numeric Applications in the Engineering at SIANI. From 2010 until now, he has been involved in several projects at SIANI and EIFER.

ENRIQUE KREMERS received his diploma in electrical engineering from the University of Karlsruhe (now: Karlsruhe Institute for Technology), Germany in 2008. Since then he has been working as a researcher at the European Institute for Energy Research in Karlsruhe in the field of modelling and simulation of energy systems. His main research focus is on agent-based modelling of complex energy systems, by developing new, integrative and bottom-up simulation approaches. $\mathrm{He}$ has contributed to several smart grid projects and is currently in charge of the agent-based modelling activities of the geo-simulation group in EIFER. 